\title{
In-situ stability of carbonates in presence of mantle phases
}

Nicole Biedermann ${ }^{1}$, Karen Appel ${ }^{1}$, Georg Spiekermann ${ }^{2}$, Wolfgang Morgenroth ${ }^{3}$, Anna Pakhomova ${ }^{4}$, Max Wilke ${ }^{2}$

${ }^{1}$ European XFEL, Schenefeld, Germany, ${ }^{2}$ Institute of Earth and Environmental Science, University of Potsdam, Potsdam, Germany, ${ }^{3}$ Institute of Geosciences, Goethe University Frankfurt, Frankfurt, Germany, ${ }^{4}$ Extreme Conditions Beamline, DESY, Hamburg, Germany

E-mail: karen.appel@xfel.eu

Understanding the processes within the Earth's lower mantle and transition zone and their physical properties as well as compositional variations is one of the grand challenges in modern geoscience. One of the large gaps concerning the Earth's lower mantle is the role of carbonates. The structure and stability of mantle carbonates has been studied intensively in the past years [1-3] and it has been shown that magnesite can be stable along the geotherm down to lower mantle conditions [3]. However, the stability of carbonates in presence of mantle silicates at relevant temperatures is far from being well understood. Related to this, very little is known about distribution processes of trace elements between carbonates and silicates.

To shed light on these processes, we studied reactions between Sr and REE-containing carbonates and relevant mantle phases (olivine, enstatite, wadsleyite and perovskite) at high pressure and temperature using synchrotron radiation based $\mu$-X-ray diffraction and $\mu$-X-ray fluorescence in a laser-heated Diamond Anvil Cell at beamline P02.2 at PETRA III. X-ray diffraction allows monitoring structural changes whereas XRF shows chemical changes. XRD and XRF maps over the laserheated spot were collected before and after the heating process. Experimental conditions reached $30 \mathrm{GPa}$ and about 2000 $K$. Single point XRD patterns recorded during heating of the assemblage allowed to observe the transformation of the starting silicates to bridgmanite ( $+\mathrm{MgO}$ in case of olivine and wadsleyite starting materials). A comparison of the XRF intensity maps before and after heating indicates a change in the elemental distribution. By superimposition of the XRFmaps to the spatial distribution of the phases as determined from additional XRD-maps, a concentration of the Sr content after annealing can be allocated around the newly formed perovskite.

Based on these experiments, we can conclude that elements are redistributed among the newly formed phases. This effect is most pronounced upon breakdown of the carbonates. If carbonate remains stable as found in one experiment, redistribution is more sluggish and could not be unequivocally constrained. Overall, it remains unclear presently to what extent these results stem from kinetic or textural effects. In any case, our first findings imply that silicates may take up the trace elements that are provided by the carbonates and indicate that carbonates may have a major effect on the trace element contents of mantle

phases by reaction.

[1] Biedermann N., Speziale S., Winkler B., Reichmann H.J., Koch-Müller M. \& Heide G. (2016) Phys Chem Minerals. DOI 10.1007/s00269-016-0861-2.

[2] Oganov A.R., Ono S., Ma Y., Glass C.W. \& Garcia A. (2008) Earth and Planetary Science Letters, 273, 38-47.

[3] Solopova N.A., Dubrovinsky L., Spivak A.V., Litvin Y.A. \& Dubrovinskaia N. (2015) Phys Chem Minerals., $42,73-81$.

Keywords: Laser-heated Diamond Anvil Cell, $\mathrm{XRD} / \mathrm{XRF}$, carbonate stability 